\title{
Microsomal prostaglandin E synthase (mPGES)-1, mPGES-2 and cytosolic PGES expression in human gastritis and gastric ulcer tissue
}

Katya Gudis $^{1}$, Atsushi Tatsuguchi ${ }^{1,2}$, Ken Wada ${ }^{1}$, Seiji Futagami ${ }^{1}$, Kazuhiro Nagata ${ }^{1}$, Tetsuro Hiratsuka ${ }^{1}$, Yoko Shinji ${ }^{1}$, Kazumasa Miyake ${ }^{1}$, Taku Tsukui ${ }^{1}$, Yuh Fukuda ${ }^{2}$ and Choitsu Sakamoto ${ }^{1}$

${ }^{1}$ Third Department of Internal Medicine, Nippon Medical School, Sendagi, Bunkyo-ku, Tokyo, Japan and

${ }^{2}$ Department of Pathology, Nippon Medical School, Sendagi, Bunkyo-ku, Tokyo, Japan

\begin{abstract}
Recently, three different prostaglandin $E_{2}$ synthases have been identified: microsomal prostaglandin $E$ synthase (mPGES)-1, cytosolic PGES (cPGES), and mPGES-2; however, their role and connection to cyclooxygenase (COX)-2 in the gastric ulcer repair process remain unknown. Therefore, we examined mPGES-1, cPGES, and mPGES-2 expression and localization in the stomach in vitro and in vivo. Tissues were obtained from Helicobacter pylori (H. pylori)-infected patients and consisted of surgical resections of gastric ulcers, or biopsies of gastric ulcers or gastritis. mPGES-1 mRNA and protein expression levels were examined by real-time polymerase chain reaction (PCR) and Western blot analysis, respectively. mPGES-1, cPGES, and mPGES-2 localization were analyzed immunohistochemically. Induction of PGES expression in response to interleukin (IL)-1 $\beta$ was examined in vitro in the cultured human gastric fibroblast line Hs262.St. Real-time PCR analysis of mPGES-1 mRNA expression in biopsy samples showed significantly higher expression levels in open than in closed gastric ulcer tissue. Western blot analysis showed mPGES-1 protein expression limited to open ulcer tissue, while mPGES-2 and cPGES immunoreactivities were seen in both open and closed ulcer tissue. Immunohistochemical analysis showed strong mPGES-1 expression in fibroblasts and macrophages of the ulcer bed, paralleling COX-2 expression. cPGES and mPGES-2 expression levels were seen in both fibroblasts of the ulcer bed and in epithelial cells. Furthermore, stronger cPGES and mPGES-2 immunoreactivities were seen in scattered mast cell-like cells and neuroendocrine-like cells, respectively. Induction of mPGES-1 expression in response to IL-1 $\beta$ was seen in cultured gastric fibroblasts in vitro, and double immunostaining showed mPGES-1 coexpression with COX-2 in fibroblasts of the ulcer bed in vivo. In conclusion, mPGES-1, cPGES, and mPGES-2 are all expressed in gastric ulcer tissue, but only mPGES-1 parallels COX-2 expression in mesenchymal and inflammatory cells of the ulcer bed, suggesting a key role for this enzyme in the ulcer repair process.
\end{abstract}

Laboratory Investigation (2005) 85, 225-236, advance online publication, 8 November 2004; doi:10.1038/labinvest.3700200

Keywords: COX-2; cPGES; gastric ulcer; mPGES-1; mPGES-2

Prostaglandin (PG) endoperoxide synthase/cyclooxygenase $(\mathrm{COX})$ is the rate limiting enzyme of $\mathrm{PG}$ synthesis from arachidonic acid. Two COX forms have been isolated; a constitutively produced COX$1^{1}$ and an inducible COX-2., ${ }^{2,3}$ Normally, COX-1 is constitutively produced in the stomach and COX-1-

Correspondence: Dr A Tatsuguchi, MD, PhD, Third Department of Internal Medicine, Nippon Medical School, 1-1-5, Sendagi, Bunkyo-ku, Tokyo 113-8603, Japan.

E-mail: tachan@nms.ac.jp

Received 18 April 2004; revised 23 September 2004; accepted 26 September 2004; published online 8 November 2004 derived $\mathrm{PGE}_{2}$ is considered involved in gastric mucosal protection, stimulating mucous bicarbonate barrier formation in the normal mucosa. ${ }^{4,5}$ Conversely, COX-2 has been shown to be induced in gastric mucosa bearing erosions or ulcers. ${ }^{6-8}$ Many studies have shown that inhibition of COX-2 activity by selective inhibitors leads to delayed healing in experimental ulcers, indicating that COX-2 induced in ulcer tissue contributes to the ulcer repair process. ${ }^{6,9}$

In addition to gastric mucosal protection, recent studies have shown that $\mathrm{PGE}_{2}$ released from gastric fibroblasts expressing COX-2 stimulates hepatocyte 
growth factor production. ${ }^{10,11}$ We have also shown that vascular endothelial growth factor (VEGF) is released from gastric fibroblasts by either interleukin (IL)-1 $\beta$ or $\mathrm{PGE}_{2}$ stimulation. ${ }^{12}$ These data suggest that, downstream of $\mathrm{COX}-2, \mathrm{PGE}_{2}$ might play an important role in epithelial cell growth as well as in the tissue repair process. $\mathrm{PGE}_{2}$ is produced from arachidonic acid via a sequence of three enzymatic reactions culminating with the isomeration of $\mathrm{PGH}_{2}$ to $\mathrm{PGE}_{2}$ by prostaglandin $\mathrm{E}$ synthase (PGES). Recently, three different PGESs have been identified; one cytosolic, cPGES, and two microsomal fractions, mPGES-1 and mPGES-2. ${ }^{13-16}$ Studies of cells overexpressing these enzymes have shown that cPGES is constitutively expressed and to be functionally coupled to COX-1 in marked preference to COX-2; ${ }^{15}$ whereas mPGES-1 has been shown to be inducible and to be preferentially coupled to COX$2,{ }^{14}$ causing a delayed $\mathrm{PGE}_{2}$ release response. mPGES-2 has yet to be well characterized, although it has been shown to be glutathione independent, ${ }^{17}$ in contrast to its sister PGESs. However, in spite of studies illustrating the mechanisms involved in $\mathrm{PGE}_{2}$ production, the contribution of each PGES to endogenous $\mathrm{PGE}_{2}$ production in gastric mucosa with gastritis or gastric ulceration has yet to be clarified. Therefore, in the present study, to better understand the role of each PGES in endogenous $\mathrm{PGE}_{2}$ production, we focused on their expression in the human gastric mucosa. Accordingly, we examined the expression of mPGES-1, cPGES, and mPGES-2 in gastric mucosa with gastritis and at various stages of ulceration to determine which PGES is actually linked to COX-2 expression in vivo. We also investigated the localization of these enzymes, and especially, whether mPGES-1 colocalizes with COX-2 in human gastric ulcer tissue.

Finally, we examined the effect of $\mathrm{IL}-1 \beta$, a proinflammatory cytokine, on mPGES-1, cPGES, and mPGES-2 expression in human gastric fibroblast cultures.

\section{Materials and methods}

\section{Patients}

Gastric tissue samples were taken from 106 Helicobacter pylori ( $H$. pylori)-infected subjects (87 males, 19 females; mean age 60 years; range, 32-82 years) with ulcers $(n=77)$ or gastritis $(n=29)$. Patients with ulcers were divided into two groups, namely open ulcers (active and healing ulcers, $n=50$ ) and closed ulcers (scarring ulcers, $n=27$ ). Three healthy individuals without $H$. pylori infection (two males, one female; mean age 58 years; range 49-70 years) were also included in the study. Samples with ulcer perforation were surgical resections from patients $(n=9)$ not undergoing treatment with $\mathrm{H}_{2}$ receptor antagonists or proton pump inhibitors. Other samples $(n=100)$ were endoscopy biopsies. Patients receiving nonsteroidal anti-inflammatory drugs for medical indications were excluded. The biopsy samples were used for immunostaining $(n=91)$, Western blot analysis $(n=6)$, and real-time polymerase chain reaction (PCR) analysis $(n=12)$. The surgical resections were used for single or double immunohistochemical analysis. All subjects gave informed consent and the project was reviewed and approved by the ethics committee of the Nippon Medical School, Tokyo, Japan.

\section{Endoscopy and Histology}

Two specimens were taken from mucosa in the gastric antrum $2 \mathrm{~cm}$ proximal to the pylorus, and one from the middle body of the greater curvature. One of the antrum specimens was used for a rapid urease test (CLO test: Delta West, Bentley, Australia) and the other one for histology. Patients infected with $H$. pylori were defined by positive results in at least two of three methods, that is, a ${ }^{13} \mathrm{C}$-urea breath test (UBT), histology, and a rapid urease test. Biopsy samples were fixed in $10 \%$ formalin fluid, dehydrated in an alcohol-xylene series, and embedded in paraffin. Sections $4 \mu \mathrm{m}$ thick were stained with hematoxylin and eosin for histological evaluation. According to the updated Sydney system, ${ }^{18}$ activity and inflammation were assessed on a scale of four grades $0,1,2$, and 3 (corresponding to none, mild, moderate, and severe). The presence of $H$. pylori in the biopsy material was determined histologically using the Giemsa stain. This evaluation was made by a single well-trained pathologist (YF) without knowledge of the endoscopic diagnosis or $H$. pylori status.

\section{Cell Line}

We used the human gastric fibroblast line Hs262.St (American Type Culture Collection; Rockville, MD, USA) for Western blot analysis of IL- $1 \beta$-induced PGE synthase expression.

\section{Antibodies}

Anti-human mouse monoclonal (MA) and guineapig polyclonal (PA) antibodies against mPGES-1 were compliments of Dr Yoshihiro Urade and $\mathrm{Dr}$ Naomi Eguchi (Osaka Bioscience Institute; Osaka, Japan), ${ }^{19}$ anti-monkey rabbit PA against mPGES- $2^{20}$ and anti-human rabbit PA against cPGES $^{15}$ were compliments of Professor Ichiro Kudo (Showa University; Tokyo, Japan). Commercial antibodies included: anti-human rabbit PA against mPGES-1 (Cayman Chemical Co.; Ann Arbor, MI, USA), antihuman rabbit PA against cPGES (Cayman), and anti-human mouse MA against COX-2 (Cayman), anti-human mouse MA against cPGES (Affinity BioReagents; Golden, CO, USA), anti-human mouse MA against chromogranin A (Dako a/s; Glostrup, Denmark), and anti-human mouse MA against CD68 
(KP1; Novocastra Laboratories Ltd.; Newcastle upon Tyne, UK).

\section{Reagents and Standards}

Recombinant human mPGES-1 protein, kindly donated by Japan Tobacco Inc., Central Pharmaceutical Research Institute; was prepared as microsomal proteins of Escherichia coli transfected with mPGES-1 cDNA. For PCR analysis, the following were used: RNeasy Mini Kit (Qiagen; Hilden, Germany), M-MLV reverse transcriptase (GIBCO Life Technologies; Gaithersburg, MD, USA), random Hexamers (Pharmacia; Freiburg, Germany), and a Taq Man probe, and $\beta$-actin primer/probe set from Applied Biosystems (Foster City, CA, USA). For Western blot, the following were used: Protein Assay Standard II kit and Precision Plus Protein Standard (Bio-Rad Laboratories; Hercules, CA, USA), Hybond polyvinylidene difluoride (PVDF) membrane and enhanced chemiluminescence (ECL) reagent (Amersham; High Wycombe, UK). Immunohistochemistry reagents were from Vector Laboratories, Inc. (Burlingame, CA, USA), except for $4^{\prime}$, 6diamidino-2-phenylindole (DAPI: Sigma Chemical; St Louis, MO, USA). For cell culture, the following were used: RPMI 1640 medium (Nikken Biomedical Laboratory; Kyoto, Japan), penicillin-streptomycin (GIBCO), fetal calf serum (FCS: Trace Biosciences PTY Ltd., New South Wales, Australia), and interleukin (IL)-1 $\beta$ (R\&D Company; Boston, MA, USA). All other reagents were of analytical grade.

\section{Real-Time PCR Analysis of mPGES-1 mRNA}

To analyze differences in mPGES-1 mRNA expression levels between ulcerated and nonulcerated tissues, we used the quantitative real-time PCR method as previously described. ${ }^{21}$ Briefly, total RNA was isolated following the RNeasy Mini Kit instructions. Reverse transcription was conducted for $60 \mathrm{~min}$ at $37^{\circ} \mathrm{C}$ using $2 \mu \mathrm{g}$ RNA in $25 \mu \mathrm{l}$ of reaction mixture, $200 \mathrm{U}$ of M-MLV reverse transcriptase and random Hexamers as primer. cDNA amplification was carried out with a sequence-detection system (GenAmp 5700). We designed the mPGES-1 primers with Primer Express software, and labeled the probe with fluorescence for the Taqman assay. The front primer was 5' -GGAACGACATGGAGACCATCTAC-3' (nucleotides 236-258); the rear primer, 5'-TCCAG GCGACAAAAGGGTTA-3' (nucleotides 300-319), with a PCR product of 84 base pairs; and the probe, 5'-FAM-TGGGCTTCGTCTACTCCTTTCT GGGTCT AMRA-3' (nucleotides 272-297). A $\beta$-actin primer/ probe set was used in separate wells as internal control for input cDNA. Thermal cycling conditions included $2 \mathrm{~min}$ at $50^{\circ} \mathrm{C}$ and $10 \mathrm{~min}$ at $95^{\circ} \mathrm{C}$, followed by 40 cycles for $15 \mathrm{~s}$ at $95^{\circ} \mathrm{C}$ and $1 \mathrm{~min}$ at $60^{\circ} \mathrm{C}$. Realtime fluorescence measurements and the cycle threshold value were determined. mPGES-1 and $\beta$-actin mRNA levels were calculated with Primer Express software. To control total cDNA input variations, mPGES-1 mRNA levels for each sample were normalized to $\beta$-actin mRNA levels and expressed as arbitrary units.

\section{Preparation of Cultured Gastric Fibroblasts}

Hs 262.St gastric fibroblasts $\left(1 \times 10^{5}\right)$ were cultured on $10 \mathrm{~cm}$ dishes in $10 \mathrm{ml}$ RPMI 1640 medium supplemented with $10 \%$ FCS and $50 \mathrm{IU} / \mathrm{ml}$ penicillin-streptomycin at $37^{\circ} \mathrm{C}$ in $5 \% \quad \mathrm{CO}_{2}$. Confluent gastric fibroblasts were then washed with phosphate-buffered saline (PBS), starved $18 \mathrm{~h}$, and cultured in the presence or absence of $10 \mu \mathrm{g} / \mathrm{ml}$ IL$1 \beta$ under serum-free conditions for $24 \mathrm{~h}$. Cells were then harvested and lysate protein content determined for Western blot analysis.

\section{Western Blot Analysis}

Gastric tissue biopsy and fibroblast samples were in turn homogenized in protein lysis/sample buffer (RIPA buffer: $50 \mathrm{mM}$ Tris, $\mathrm{pH}$ 8.0, $0.1 \%$ sodium dodecyl sulfate, $1 \%$ Nonidet P-40, $0.5 \%$ deoxycholate, $150 \mathrm{mM} \mathrm{NaCl}, 1 \mathrm{mM}$ PMSF), stored in ice for $15 \mathrm{~min}$, centrifuged at $15000 \mathrm{rpm} 20 \mathrm{~min}$ and the resultant supernatants used as protein lysis samples. Recombinant mPGES-1 protein was used as positive control. Proteins were solubilized in sodium dodecyl sulfate (sample buffer), separated on $15 \%$ sodium dodecyl sulfate-polyacrylamide gels, subjected to electrophoresis, and transferred onto Hybond PVDF membranes. Membranes were blocked overnight at $4^{\circ} \mathrm{C}$ in $5 \%$ powdered milk in Tris-buffered saline Tween (TBST), then incubated with mPGES-1 PA (Cayman; dilution, 1:500) or mPGES-2 PA (Showa; dilution, 1:2000) in TBST for $1 \mathrm{~h}$. In addition, membranes with biopsy proteins were also incubated with cPGES MA (ABR; dilution, 1:1000), and those with fibroblast lysates with CPGES PA (Cayman; 1:500). Blots were washed with TBST and probed with anti-mouse or anti-rabbit horseradish peroxidase-conjugated secondary antibody at concentrations of 1:2000 for $1 \mathrm{~h}$, and finally developed with ECL reagent.

\section{Single Immunohistochemical Analysis}

Gastric tissue biopsy samples, from either the ulcer edge or corpus of the stomach, and surgically resected gastric ulcer tissues with perforation were used for immunohistochemical analysis of COX-2, mPGES-1, mPGES-2, and cPGES. Specimens were fixed in $10 \%$ formalin, embedded in paraffin wax, cut into $3 \mu \mathrm{m}$ sections, and immersed in $0.3 \% \mathrm{H}_{2} \mathrm{O}_{2}{ }^{-}$ methanol for $30 \mathrm{~min}$ to block endogenous peroxidase activity. Sections were then microwaved in $0.01 \mathrm{~mol} / \mathrm{l}$ citrate phosphate buffer $(\mathrm{pH}$ 6.0) for antigen retrieval, and incubated with $10 \%$ normal 
horse or goat serum for $20 \mathrm{~min}$ at $37^{\circ} \mathrm{C}$ to block nonspecific immunoglobulin (IgG) binding. Sections were then incubated for $20 \mathrm{~h}$ at $4^{\circ} \mathrm{C}$ with either mouse anti-human COX-2 (diluted 1:200), mouse anti-human mPGES-1 (diluted 1:100 000), rabbit anti-monkey mPGES-2 (diluted 1:1000) or rabbit anti-human cPGES (diluted 1:1000), and then incubated for $1 \mathrm{~h}$ at $37^{\circ} \mathrm{C}$ with their respective biotinilated anti-mouse or anti-rabbit IgG (1:200). Antibody binding sites were visualized by treating sections with avidin biotinilated peroxidase complex for $1 \mathrm{~h}$ at $37^{\circ} \mathrm{C}$, then immersing sections in $3,3^{\prime}-$ diaminobenzidine tetrahydrochloride solution containing $0.03 \% \quad \mathrm{H}_{2} \mathrm{O}_{2}$. Nuclei were counterstained with Mayer's hematoxylin. mPGES-1 antibody specificity was determined by preadsorption of the primary antibody with human mPGES-1 protein for $2 \mathrm{~h}$ at $37^{\circ} \mathrm{C}$ prior to staining procedures.

\section{Scoring Methods}

Immunohistochemical findings in biopsy specimens were analyzed according to ulcer stage. We prepared at least two sections from each biopsy sample to test for mPGES-1, mPGES-2, and cPGES positivity. Most specimens contained surface epithelium and muscularis mucosa. When immunoreactivity was observed in at least one mesenchymal or inflammatory cell, patients were classified as positive for mPGES1. Negative cases consisted of sections with complete lack of mesenchymal or inflammatory cell immunostaining. When immunoreactivity was observed in at least one mesenchymal or glandular epithelial cell, patients were classified as positive for mPGES-2 or cPGES. All sections were examined independently by an expert pathologist blind to pathological and clinical data.

\section{Double Immunohistochemical Analysis}

Double labeling using immunofluorescence methods and confocal laser scanning microscopy was used to evaluate colocalization of immunoreactivity for the following paired antibodies: mPGES-1 (PA)/ COX-2 (MA), mPGES-2 (PA)/CD68 (MA), and cPGES (PA)/chromogranin A (MA). Each primary antibody pair was applied to separate surgically resected gastric ulcer tissue samples and incubated overnight at $4{ }^{\circ} \mathrm{C}$. The primary MA against COX-2, CD68, and chromogranin A was allowed to react with a secondary antibody (horse anti-mouse IgG; dilution, 1:100 for the mPGES-1/COX-2 pair, 1:50 for the others) labeled with Texas red. The primary PAs were allowed to react with their respective secondary antibodies: mPGES-1 (PA) with fluorescein isothiocynate (FITC) labeled anti-guinea-pig IgG (dilution, 1:100), and mPGES-2 (PA) and CPGES (PA) with FITC labeled anti-rabbit IgG (dilution, 1:50). The nuclei were then counterstained with DAPI and tissues examined with a confocal micro- scope (model TCS4D/DMIRBE; Leica, Heidelberg, Germany) equipped with argon and argon-krypton laser sources.

\section{Statistical Analysis}

Statistical analysis was performed with Statview software (Abacus Concept Inc.; Berkeley, CA, USA). The $\chi^{2}$ for $2 \times 2$ tables was used with the Fisher's exact test to analyze significant differences between immunohistochemical groups. The correlations among inflammation, activity, atrophy, metaplasia, and $H$. pylori score with each PGES immunoreactivity were evaluated by Spearman's rank correlation test. The Mann-Whitney $U$-test was used to analyze significant differences in real-time PCR data. A value of $P<0.05$ indicated statistically significant differences.

\section{Results}

\section{mPGES-1 mRNA Expression Levels in Gastric Tissue}

We first determined mPGES-1 mRNA expression levels in human gastric mucosa with open or closed ulcers by quantitative real-time PCR. mPGES-1 and $\beta$-actin mRNA expressions were examined using biopsy samples from open ulcers $(n=6)$ and closed ulcers $(n=6)$ and detected in all samples tested. mPGES-1/ $\beta$-actin mRNA ratios were significantly higher in open ulcerated than in closed ulcerated tissue (Figure 1; $P<0.05$ ).

\section{mPGES-1, mPGES-2, and cPGES Protein Expression Levels in Gastric Tissue}

mPGES-1 protein expression in gastric ulcer tissue was observed as a band of $16 \mathrm{kDa}$ corresponding to

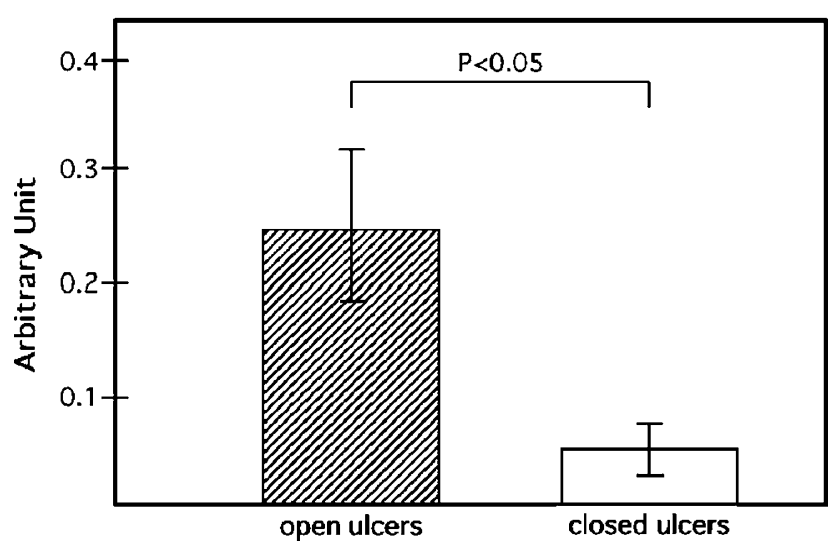

Figure 1 Real-time PCR analysis of mPGES-1 mRNA expression in gastric tissue with open and closed ulcers. Total RNA was isolated from biopsy samples taken from gastric mucosa with open and closed ulcers (each, $n=6$ ) and real-time RT-PCR performed as described under Materials and methods. This is a representative of two independent experiments performed with three biopsy samples in each group. Data are expressed as means \pm s.e. $P<0.05$, Mann-Whitney $U$-test. 


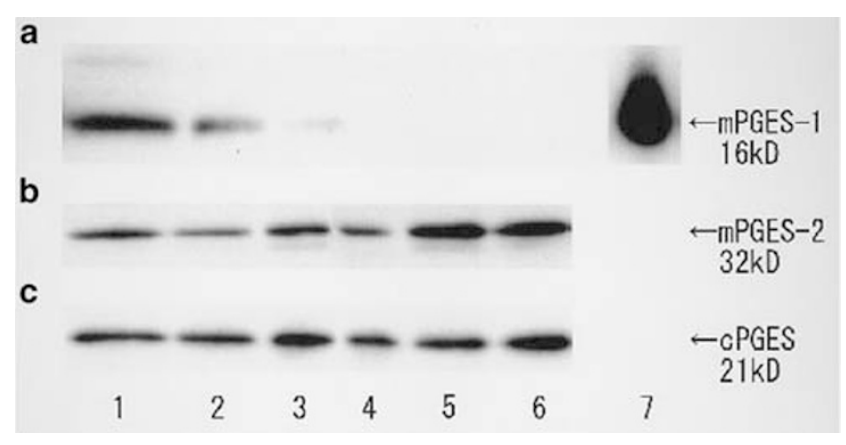

Figure 2 Gastric tissue samples were lysed, and lysates subjected to electrophoresis as described under materials and methods. Separated proteins on Hybond PVDF membranes were probed with (a) anti-mPGES-1 antibody, (b) anti-mPGES-2 antibody, or (c) anti-cPGES antibody. The lanes are as follows: 1-3, open ulcer; 4, closed ulcer; 5-6, gastritis; 7 , recombinant mPGES-1 protein.

the recombinant mPGES-1 protein, our positive control (Figure 2a). mPGES-1 protein was seen only in samples from the open ulcer margin, with no expression detected in samples from closed ulcers or gastritis mucosa. mPGES-2 and cPGES protein were observed as bands of 32 and $21 \mathrm{kDa}$, closely corresponding to molecular sizes previously reported $^{15,16}$ (Figure 2b,c). In contrast to mPGES-1, mPGES-2 and CPGES protein expression levels were not limited to gastric tissue samples of the open ulcer margin, but were also expressed in samples of the closed ulcer and gastritis mucosa.

\section{Localization of mPGES-1, mPGES-2, and cPGES in Gastric Tissue}

We next examined the localization of mPGES-1, mPGES-2, and cPGES proteins immunohistochemically and compared it with COX-2 localization in gastric mucosa with ulceration or gastritis. Immunohistochemical analysis of surgical resections of ulcerated tissue showed strong COX-2 expression in mesenchymal and inflammatory cells, corresponding to fibroblasts and macrophages, in granulation tissue of the ulcer bed (Figure 3a), with weaker expression seen in epithelial cells but only at the ulcer edge (Figure 3b). As in COX-2, mPGES-1 protein expression was observed in mesenchymal and inflammatory cells of the ulcer bed (Figure 3c, e). We also found weak mPGES-1 expression in epithelial cells, but again, only at the ulcer edge (Figure 3d); epithelial cells far from the ulcer edge and gastritis mucosa failed to express any mPGES-1 (Figure 3f). In contrast, cPGES and mPGES-2 protein expression levels were observed not only in mesenchymal cells of the ulcer bed (Figure 4a, b, f, g) but also in epithelial cells of gastritis mucosa (Figure $4 c, d, i, j)$. cPGES expression was observed in fibroblasts and inflammatory cells like macrophages, similar to mPGES-1. In addition, endothelial cells also showed fairly strong cPGES immunoreactivity (Figure 4b). Of interest, the strongest cPGES im- munoreactivity was seen in endocrine-type epithelial cells (Figure 4d, e), with rather weak expression of glandular epithelial cells in gastritis mucosa (Figure 4c). mPGES-2 was also observed in fibroblasts of the ulcer bed (Figure 4f,g) and glandular epithelial cells at the ulcer edge (Figure 4i) and gastritis mucosa (Figure 4j), while the strongest mPGES-2 immunoreactivity was seen in spherical cells resembling macrophages or mast cells, scattered around the ulcer bed. These results suggest that cPGES and mPGES-2 seem to have their own specific functions in different cells, in addition to their being involved in mesenchymal and epithelial cell PG production.

We then compared mPGES-1, cPGES, and mPGES2 expression levels at different stages of ulceration. The positive rates of mPGES-1 immunoreactivity were significantly higher in open ulcers than in closed ulcers (Table 1, $P<0.05$ ). cPGES and mPGES2 immunoreactivities did not differ between open and closed ulcers.

We next compared mPGES-1, cPGES, and mPGES2 expressions in gastritis mucosa and normal mucosa without gastritis. The clinicopathological data of these subjects are shown in Table 2. In the $H$. pylori negative group $(n=3)$, no mPGES-1 immunoreactivity was noted both in epithelial cells or lamina propria mesenchymal cells. mPGES-1 immunoreactivity was found only in mesenchymal and inflammatory cells in nine out of $27 \mathrm{H}$. pylorirelated gastritis samples. Among all Sydney system test scores for patients infected with $H$. pylori, a correlation was seen between mPGES-1 immunoreactivity and inflammation/activity/H. pylori grade $(r=0.368, \quad P=0.0475 ; \quad r=0.582, \quad P=0.0017 ; \quad r=$ $0.612, P=0.001$, respectively, Spearman rank correlation test), but not atrophy/metaplasia grade (Table 3). mPGES-2 immunoreactivity was detected in two out of three $H$. pylori negative groups, and 24 out of $27 \mathrm{H}$. pylori positive groups. There was an inverse correlation between mPGES-2 immunoreactivity and atrophy grade $(r=-0.368, P=0.047)$. cPGES immunoreactivity was detected in two out of three $\mathrm{H}$. pylori negative groups, and 22 out of $27 \mathrm{H}$. pylori positive groups. There was no relationship between cPGES immunoreactivity and any scores of the Sydney system.

\section{mPGES-1, cPGES, and mPGES-2 Expression in Cultured Gastric Fibroblasts}

As we saw all three PGE synthases expressed in spindle-shaped mesenchymal cells of the ulcer bed, we next examined whether all these enzymes could also be seen and induced in cultured gastric fibroblasts in vitro. mPGES-1 expression was observed as a $16 \mathrm{kDa}$ protein, exactly corresponding to the molecular weight seen for gastric ulcer tissue samples, and its expression level increased following IL-1 $\beta$ stimulation (Figure 5a). Molecular weights 

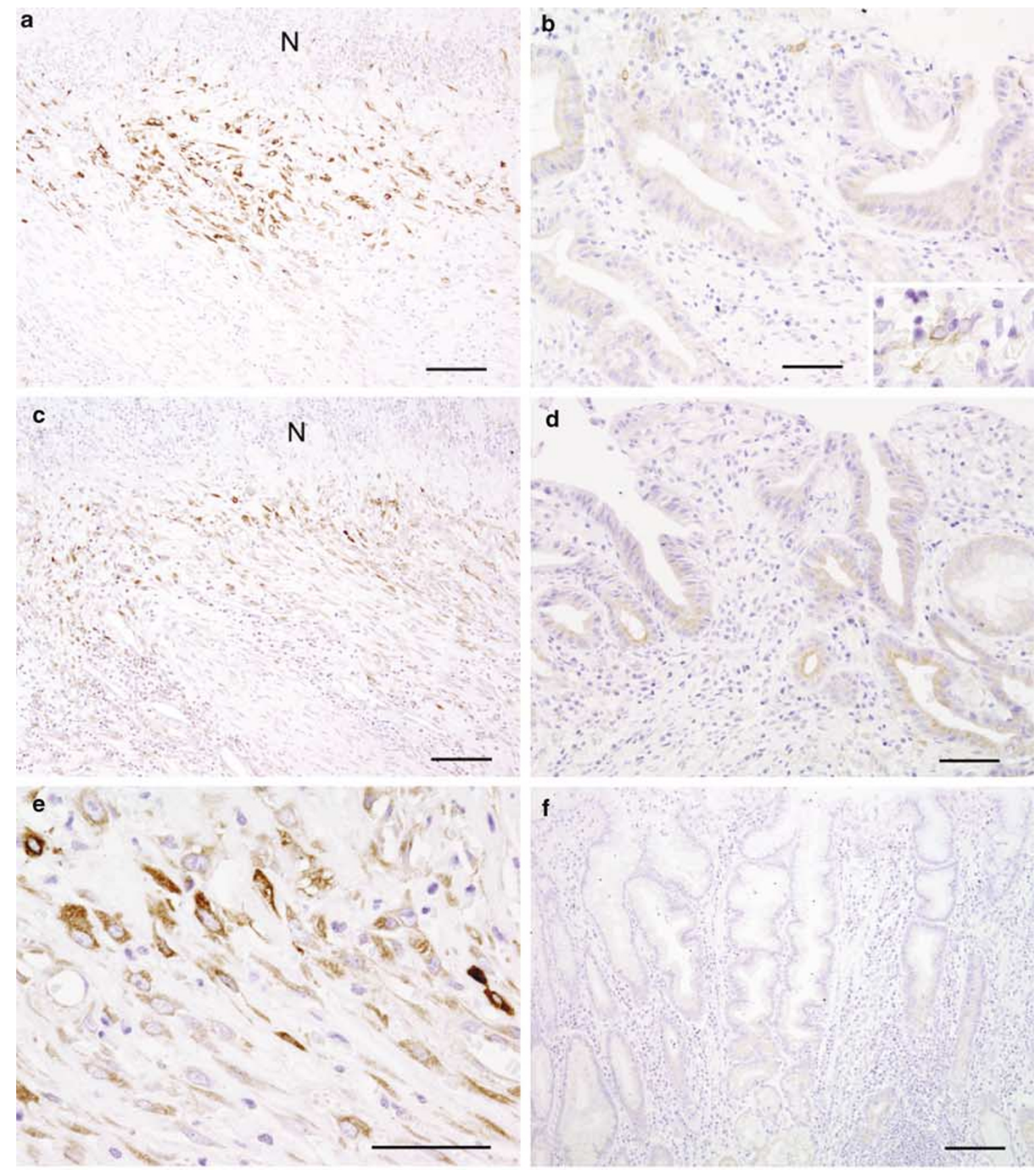
for cPGES and mPGES-2 protein expression, respectively observed as 21 and $32 \mathrm{kDa}$ proteins, also corresponded in $\mathrm{kDa}$ values with their tissue sample counterparts. However, in contrast to mPGES-1 expression, cPGES and mPGES-2 protein expression levels did not change following IL-1 $\beta$ stimulation (Figure 5b,c).

\section{COX-2 and mPGES-1 Coexpression in Gastric Ulcer Tissue}

Numerous inflammatory and spindle-shaped cells of the ulcer bed expressed mPGES-1 immunoreactivity (Figure 6a). Strong COX-2 immunoreactivity was also observed in inflammatory and mesenchymal cells of the same ulcer bed section (Figure 6b). Double immunostaining of mPGES-1 and COX-2 with immunofluorescence conjugating antibodies revealed that mPGES-1 and COX-2 were coexpressed in spindle-shaped cells of the ulcer bed (Figure 6c).

\section{Characterization of cPGES and mPGES-2 Positive Cells in Gastric Tissue}

Clear cPGES immunoreactivity was seen in glandular epithelial cells in gastric tissue (Figure 6d). Chromogranin A immunoreactivity was also observed in the same section of glandular epithelium (Figure 6e) and double immunostaining clearly showed that cPGES immunoreactivity colocalized with chromogranin A immunoreactivity (Figure 6f). mPGES-2 immunoreactivity in spherical cells was scattered in granulation tissue of the ulcer bed (Figure 6g). Although macrophages were also observed in the same section of the gastric ulcer bed (Figure 6h), double immunostaining of mPGES-2 and CD68 suggests that mPGES-2 immunoreactivity was not localized in macrophages (Figure 6i).

\section{Discussion}

We describe here, for the first time, mPGES-1 mRNA and protein expression in human gastric ulcer tissue. Real-time PCR analysis and Western blot analysis of gastric ulcer tissue clearly showed strong mPGES-1 gene and protein expression levels in open ulcers, lower mPGES-1 protein expression levels in closed ulcers, and no expression, whatso- ever, in gastritis mucosa. Furthermore, in the present study, mPGES-1 enzyme was strongly expressed in macrophages and fibroblasts found exclusively between granulation and necrotic tissue of and around the gastric ulcer bed. This location corresponds precisely with that found for COX-2 expression in our previous study on the human stomach. ${ }^{8}$ Confocal double immunostaining of COX2 and mPGES-1 confirmed mPGES- 1 and COX-2 colocalization in fibroblast-like cells infiltrating into granulation tissue of the ulcer bed, raising the possibility that $\mathrm{PGE}_{2}$ is released in those cells. In addition, in gastric fibroblasts cultured in vitro, we found that mPGES-1 expression was induced by the proinflammatory cytokine IL- $1 \beta$, as we have recently shown for COX-2 expression in gastric fibroblasts. ${ }^{12}$ Thus, our immunohistochemical analysis and in vitro data support possible functional coupling between mPGES-1 and COX-2 in mesenchymal cells of ulcerogenic gastric mucosa, in line with recent in vitro and in vivo studies showing coexpression of these enzymes. ${ }^{22-26}$ In cells cotransfected with mPGES-1 and COX-2 genes, synergetic COX-2 and mPGES-1 expression levels were shown to be crucial for efficient $\mathrm{PGE}_{2}$ production. ${ }^{14,27}$ In addition to these isolated cell systems, mPGES-1 has been shown to be essential for $\mathrm{PGE}_{2}$ production in the inflammatory response: mPGES-1 was expressed in the hind paws of rats with adjuvant arthritis ${ }^{28}$ and in synoviocytes from patients with rheumatoid arthritis. ${ }^{29,30}$ In a previous study, we were able to demonstrate the crucial role of COX-2 in the ulcer repair process, ${ }^{6}$ suggesting that $\mathrm{PGE}_{2}$ release subsequent to mPGES-1 expression might also be involved in tissue repair.

In the present study, we saw weak mPGES-1 expression in gastric epithelial cells, although only at the ulcer edge, exactly paralleling COX-2 expression. In a previous study, we reported that COX-2 immunoreactivity was found exclusively in stromal cells, such as macrophages and fibroblasts, in $H$. pylori-related gastritis and gastric ulcer tissue. ${ }^{8} \mathrm{We}$ could find no constitutive expression of COX-2 in epithelial cells other than in those with regenerative changes at the edge of ulcers and intestinal metaplasia. Both our previous and current studies are in sharp contrast to those showing COX-2 expression in gastric epithelial cells in vitro ${ }^{31,32}$ and in vivo. ${ }^{33-36}$ For example, in vitro studies show that $H$. pylori induces COX-2 expression in gastric

Figure 3 Immunohistochemical localization of COX-2 and mPGES-1 in gastric ulcer tissue. (a) Low magnification view of the ulcer bed. Necrotic tissue $(\mathrm{N})$ covered surface of ulcerated area. COX-2 was strongly stained in many mesenchymal cells or mononuclear cells beneath necrotic tissue $(\mathrm{N})$ of the ulcer bed. (b) COX-2 was stained in scattered lamina propria mesenchymal cells of the ulcer edge. Inset: higher magnification of lamina propria mesenchymal cells. (c) The same area as (a). mPGES-1 was strongly stained in many mesenchymal cells or mononuclear cells beneath necrotic tissue $(\mathrm{N})$ of the ulcer bed. (d) The same area as (b). mPGES-1 showed a fair amount of staining in regenerating epithelial cells of the ulcer edge. (e) Higher magnification view of (c). (f) Gastritis mucosa away from the ulcer bed. mPGES-1 immunoreactivity was not significant throughout gastritis mucosa. (g) The same area as (a). Control specimen, treated with mPGES blocking peptide and primary antibody, showed no reaction. N, necrotic tissue. Bars: a, c, f, g=100 $\mu$ m; b, d, $\mathbf{e}=50 \mu \mathrm{m}$. 

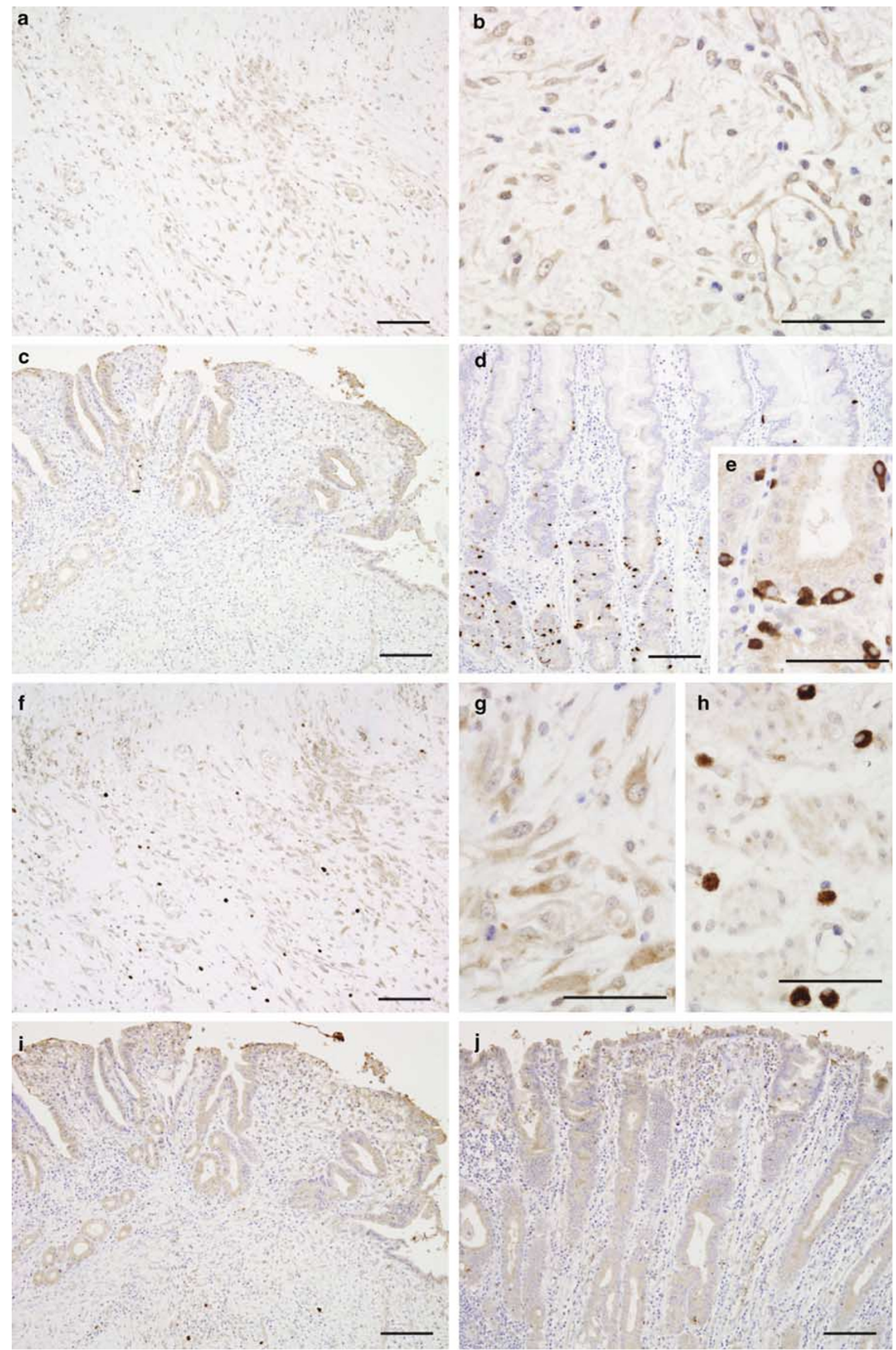
epithelial cells. ${ }^{31}$ There are also in vivo reports showing that COX-2 is expressed in the epithelial cells rather than in mesenchymal and inflammatory cells in $H$. pylori gastritis tissue. ${ }^{33-35}$ In addition, Nardone et $a l^{36}$ have recently reported that not only COX-2 but also mPGES-1 expression were seen both

Table 1 Comparison of positive rates of PGES immunoreactivities between open and closed ulcers

\begin{tabular}{lccc}
\hline & $\begin{array}{c}\text { mPGES-1 } \\
\text { positive (\%) }\end{array}$ & $\begin{array}{c}\text { cPGES } \\
\text { positive (\%) }\end{array}$ & $\begin{array}{c}\text { mPGES-2 } \\
\text { positive (\%) }\end{array}$ \\
\hline Open ulcers $(n=32)$ & $20(63)$ & $26(81)$ & $27(84)$ \\
Closed ulcers $(n=20)$ & $2(10)^{*}$ & $17(85)$ & $16(80)$ \\
\hline
\end{tabular}

${ }^{*} P<0.001$ vs PGES-1 positive rates at open ulcers.

Table 2 Clinical and average score of the Sydney system parameters of $H$. pylori negative and positive subjects

\begin{tabular}{lcc}
\hline & $\begin{array}{c}\text { H. pylori negative } \\
\text { subjects }(n=3)\end{array}$ & $\begin{array}{c}\text { H. pylori positive } \\
\text { gastritis }(n=27)\end{array}$ \\
\hline Age (years) & $54 \pm 6$ & $60 \pm 2$ \\
Sex (M/F) & $2 / 1$ & $25 / 2$ \\
& & \\
Sydney system parameters & $1 \pm 0$ & $2.3 \pm 0.1$ \\
Inflammation & 0 & $2.4 \pm 0.2$ \\
Activity & $0.5 \pm 0.3$ & $0.6 \pm 0.2$ \\
Atrophy & $1.3 \pm 0.3$ & $1.7 \pm 0.2$ \\
Metaplasia & 0 & $2.3 \pm 0.2$ \\
H. pylori & & \\
\hline
\end{tabular}

in epithelial and stromal cells of $H$. pylori-related chronic active gastritis and intestinal metaplasia. This conflicting data regarding localization of COX2 and mPGES-1 proteins may be due in part to nonspecific binding of polyclonal antibodies. It is

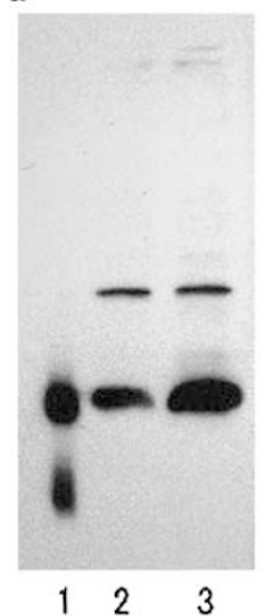

b

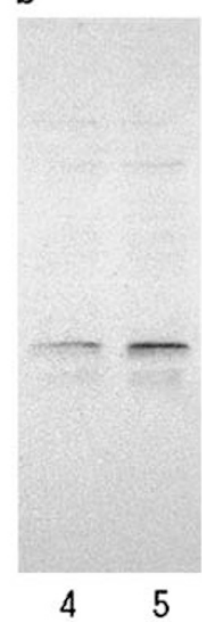

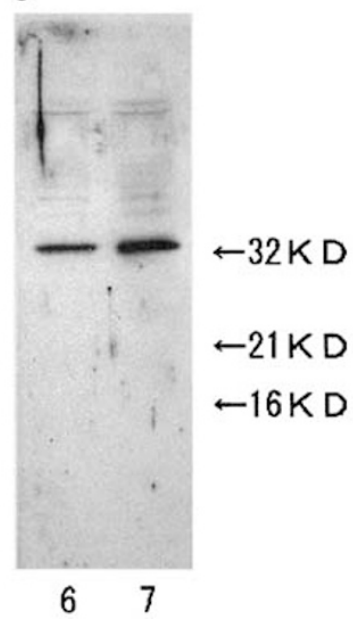

Figure 5 Western blot analysis of mPGES-1, cPGES, and mPGES-2 expression levels in cultured gastric fibroblasts with or without IL-1 $\beta$ stimulation. Gastric fibroblasts cultured in serum-free medium were incubated in the presence or absence of IL-1 $\beta$ at $10 \mu \mathrm{g} / \mathrm{ml}$ for $24 \mathrm{~h}$. The fibroblasts were then lysed, samples electrophoresed, and proteins separated on Hybond PVDF membranes probed with (a) anti-mPGES-1 antibody, (b) anticPGES antibody, or (c) anti-mPGES-2 antibody. The lanes are as follows: 1, recombinant mPGES-1 protein; 2, 4 and 6, gastric fibroblasts without IL-1 $\beta$ stimulation; $3,5,7$, gastric fibroblasts with IL-1 $\beta$ stimulation.

Table 3 Relationship between PGES expression in H. pylori-related gastritis and scores of the Sydney system parameters

\begin{tabular}{|c|c|c|c|c|c|c|}
\hline & \multicolumn{2}{|c|}{ mPGES-1 } & \multicolumn{2}{|c|}{ cPGES } & \multicolumn{2}{|c|}{$m P G E S-2$} \\
\hline & $\mathrm{r}$ & $\mathrm{P}$ & $\mathrm{r}$ & $\mathrm{P}$ & $\mathrm{r}$ & $\mathrm{P}$ \\
\hline Inflammation & 0.368 & 0.048 & 0 & NS & 0.093 & NS \\
\hline Activity & 0.582 & 0.002 & 0.201 & NS & 0.050 & NS \\
\hline Atrophy & -0.259 & NS & -0.145 & NS & -0.368 & 0.047 \\
\hline Metaplasia & -0.185 & NS & -0.302 & NS & -0.142 & NS \\
\hline H. pylori & 0.612 & 0.001 & 0.203 & NS & 0.018 & NS \\
\hline
\end{tabular}

NS, not significant.

Figure 4 Immunohistochemical localization of cPGES and mPGES-2 in gastric ulcer tissue. (a) Low magnification view of the ulcer bed. cPGES was stained in many mesenchymal cells or mononuclear cells beneath necrotic tissue (N) of the ulcer bed. (b) Higher magnification view of (a). cPGES was stained in many mesenchymal cells or mononuclear cells as well as in endothelial cells. (c) cPGES showed a fair amount of staining in regenerating epithelial cells of the ulcer edge. (d) cPGES was strongly stained in endocrine cells from the neck to the base of crypts in gastric mucosa away from the ulcer bed. (e) Higher magnification view of (d). cPGES positive cells were present beneath epithelial cells as closed-type neuroendocrine-like cells. (f) Low magnification view of the ulcer bed. mPGES-2 was stained in many mesenchymal cells or mononuclear cells beneath necrotic tissue $(\mathrm{N})$ of the ulcer bed. (g) Higher magnification view of (f). mPGES-2 was stained in many mesenchymal cells. (h) Higher magnification view of (f). mPGES-2 was strongly stained in many mononuclear cells distributed in granulation tissue. (i) mPGES-2 showed a fair amount of staining in regenerating epithelial cells of the ulcer edge. (j) mPGES-2 was stained in glandular epithelial cells in gastric mucosa away from the ulcer bed. N, necrotic tissue. Bars: a, c, $\mathbf{d}, \mathbf{f}, \mathbf{i}, \mathbf{j}=100 \mu \mathrm{m} ; \mathbf{b}, \mathbf{e}, \mathbf{g}, \mathbf{h}=50 \mu \mathrm{m}$. 

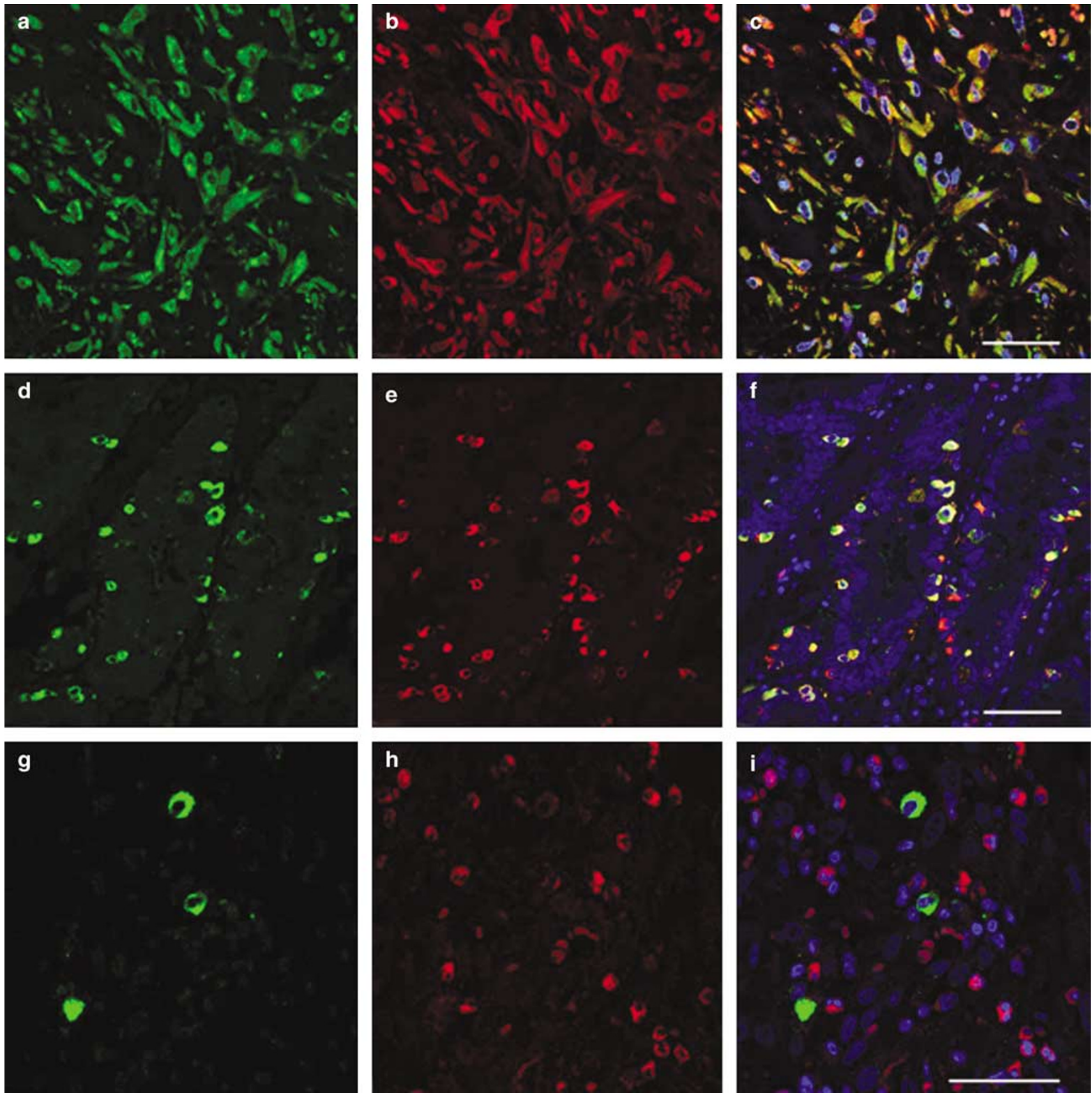

Figure 6 Confocal microscopic images of sections stained by dual labeling procedures, using FITC and Texas red-conjugated antibodies. Colocalization is shown in yellow. Nuclei were counterstained with DAPI (blue fluorescence). (a) mPGES-1 (green) was strongly stained in many mesenchymal cells or mononuclear cells beneath necrotic tissue of the ulcer bed. (b) COX-2 (red) was strongly stained in many mesenchymal cells or mononuclear cells beneath necrotic tissue of the ulcer bed. (c) Merging panels a and $\mathbf{b}$ reveal that all mesenchymal cells or mononuclear cells exhibit colocalization (yellow) of these proteins. (d) cPGES (green) was strongly stained in endocrine cells from the neck to the base of crypts in gastric mucosa. (e) Chromogranin A (red) was strongly stained in endocrine cells from the neck to the base of crypts in gastric mucosa. (f) Merging panels $\mathbf{d}$ and $\mathbf{e}$ reveal that cPGES positive cells are completely identical to chromogranin A positive cells. (g) mPGES-2 (green) was strongly stained in a few mononuclear cells distributed in granulation tissue. (h) CD68 positive cells were diffusely distributed in granulation tissue. (i) Merging panels $\mathbf{g}$ and $\mathbf{h}$ reveal that mPGES-2 positive cells are different from CD68 positive cells. Bars $=50 \mu \mathrm{m}$.

now clear that mPGES-1 is an inducible enzyme and actually upregulated in response to various stimuli, thereby acting downstream for COX-2-derived $\mathrm{PGE}_{2}$ generation. Thus, it makes sense that mPGES-1 and COX-2 are colocalized in the same cells. Further- more, this colocalization of mPGES-1 and COX-2 in inflammatory and interstitial cells, in the present study, reflects the crucial role of endogenous $\mathrm{PGE}_{2}$ in VEGF production in gastric fibroblasts during the ulcer repair process in the human stomach. 
In the present study, we also examined cPGES and mPGES-2 expression levels in gastritis and ulcerated gastric mucosa. Western blot analysis of cPGES protein showed equal expression levels not only for intact and ulcerated mucosa but also for unstimulated and IL- $1 \beta$-stimulated cultured gastric fibroblasts, consistent with previous studies showing that with respect to $\mathrm{PGE}_{2}$ production, cPGES enzyme is linked to constitutive COX-1. ${ }^{14}$ In addition to mesenchymal cells of the ulcer bed, cPGES immunoreactivity was also found in epithelial cells. Among epithelial cells expressing cPGES, very strong immunoreactivity was observed in certain endocrine-type cells. Confocal double immunostaining clearly shows that CPGES colocalized with chromogranin A immunoreactivity, suggesting that cPGES might play a key role in $\mathrm{PGE}_{2}$ production in gastric epithelial endocrine cells. mPGES-2 was also uniformly expressed, both in gastritis and ulcerated gastric mucosa, and in gastric fibroblasts in vitro, in the presence or absence of IL- $1 \beta$, suggesting constitutive expression of mPGES-2, analogous to cPGES expression. ${ }^{20,28}$ However, strong immunoreactivity was also seen for mPGES-2 in spherical cells scattered around the ulcer bed, compared to rather weak expression in epithelial and other interstitial cells, suggesting a yet to be determined role for mPGES-2 in those cells. Confocal double immunostaining data show that this strong mPGES2 immunoreactivity was not localized in macrophages. Thus, to identify this particular cell type expressing strong mPGES-2, further work is required.

Although it is now well known that selective COX-2 inhibitors are very effective therapeutics for decreasing joint pain and for chemoprevention of polyp tumor progression in familial adenomatous polyposis patients, ${ }^{37}$ recent studies have shown that long-term use of these highly selective COX-2 inhibitors might increase the risk of cardiovascular events, suggesting that selective COX-2 inhibitors might alter the balance between COX-1-derived thromboxane $\mathrm{A}_{2}$ and COX-2-derived PG production. $^{38}$ Thus, a more selective modulation of the $\mathrm{PGE}_{2}$ pathway appears to be desirable, raising the possibility of mPGES-1 as an attractive novel target for therapeutic intervention. ${ }^{13}$ However, if mPGES1-derived $\mathrm{PGE}_{2}$ plays a role in the ulcer repair process as a downstream signal of COX-2, or in other physiological roles related to COX-2 induction, as has been shown for ovulation and renal salt transport, ${ }^{39,40}$ it is possible that any drug specifically targeting mPGES-1 may not only delay ulcer healing but also interfere with the physiological role of $\mathrm{PGE}_{2}$.

Clearly, further work is necessary to determine whether mPGES-1-derived $\mathrm{PGE}_{2}$ plays a key role in the ulcer repair process and whether mPGES-1specific antagonists can in the future prove to be neither ulcerogenic nor to delay ulcer healing in humans.

\section{Acknowledgements}

We thank Drs Yoshihiro Urade and Naomi Eguchi (Osaka Bioscience Institute; Osaka, Japan) for kindly supplying antibody mPGES-1, Professor Ichiro Kudo (Showa University; Tokyo, Japan) for kindly donating antibodies mPGES-2 and cPGES; and Japan Tobacco Inc., Central Pharmaceutical Research Institute for providing mPGES-1 protein, and Mrs Hideko Yamagishi for technical assistance.

\section{References}

1 Dewitt DL, Smith WL. Primary structure of prostaglan$\operatorname{din} \mathrm{G} / \mathrm{H}$ synthase from sheep vesicular gland determined from the complementary DNA sequence. Proc Natl Acad Sci USA 1988;85:1412-1416.

2 Hla T, Neilson K. Human cyclooxygenase-2 cDNA. Proc Natl Acad Sci USA 1992;266:7384-7388.

3 Pritchard Jr KA, O’Banion MK, Miano JM, et al. Induction of cyclooxygenase-2 in rat vascular smooth muscle cells in vitro and in vivo. J Biol Chem 1994; 269:8504-8509.

4 Robert A, Nezamis JE, Lancaster C, et al. Cytoprotection by prostaglandins in rat. Prevention of gastric necrosis produced by alcohol, HCL, hypertonic $\mathrm{NaCl}$, and thermal injury. Gastroenterology 1979;77:433-443.

5 Terano A, Ota S, Mach T, et al. Prostaglandin protects against taurocholate-induced damage to rat gastric mucosal cell culture. Gastroenterology 1987;92: 669-677.

6 Mizuno H, Sakamoto C, Matsuda K, et al. Induction of cyclooxygenase 2 in gastric mucosal lesions and its inhibition by the specific antagonist delays healing in mice. Gastroenterology 1997;112:387-397.

7 Takahashi S, Shigeta J, Inoue H, et al. Localization of cyclooxygenase-2 and regulation of its mRNA expression in gastric ulcers in rats. Am J Physiol Gastrointest Liver Physiol 1998;275:G1137-G1145.

8 Tatsuguchi A, Sakamoto C, Wada K, et al. Localisation of cyclooxygenase 1 and cyclooxygenase 2 in Helicobacter pylori related gastritis and gastric ulcer tissues in humans. GUT 2000;46:782-789.

9 Jones MK, Wang H, Peskar BM, et al. Inhibition of angiogenesis by nonsteroidal anti-inflammatory drugs: insight into mechanisms and implications for cancer growth and ulcer healing. Nat Med 1999;5:1418-1423.

10 Takahashi M, Ota S, Shimada T, et al. Hepatocyte growth factor is the most potent endogenous stimulant of rabbit gastric epithelial cell proliferation and migration in primary culture. J Clin Invest 1995;95: 1994-2003.

11 Bamba H, Ota S, Kato A, et al. Nonsteroidal antiinflammatory drugs may delay the repair of gastric mucosa by suppressing prostaglandin-mediated increase of hepatocyte growth factor production. Biochem Biophys Res Commun 1998;245:567-571.

12 Miura S, Tatsuguchi A, Wada K, et al. Cyclooxygenase2-regulated vascular endothelial growth factor release in gastric fibroblasts. Am J Physiol Gastrointest Liver Physiol 2004;287:G444-G451.

13 Jakobsson PJ, Thorén S, Morgenstern R, et al. Identification of human prostaglandin E synthase: a microsomal glutathione-dependent, inducible enzyme, 
constituting a potential novel drug target. Proc Natl Acad Sci USA 1989;96:7220-7225.

14 Murakami M, Naraba H, Tanioka T, et al. Regulation of prostaglandin E2 biosynthesis by inducible membraneassociated prostaglandin E2 synthase that acts in concert with cyclooxygenase-2. J Biol Chem 2000; 275:32783-32792.

15 Tanioka T, Nakatani Y, Semmyo N, et al. Molecular identification of cytosolic prostaglandin E2 synthase that is functionally coupled with cyclooxygenase- 1 in immediate prostaglandin E2 biosynthesis. J Biol Chem 2000;275:32775-32782.

16 Tanikawa N, Ohmiya Y, Ohkubo H, et al. Identification and characterization of a novel type of membraneassociated prostaglandin E synthase. Biochem Biophys Res Commun 2002;291:884-889.

17 Watanabe K, Kurihara K, Tokunaga Y, et al. Two types of microsomal prostaglandin E synthase: glutathionedependent and -independent prostaglandin E synthases. Biochem Biophys Res Commun 1997;235: 148-152.

18 Dixon MF, Genta RM, Yardley JH, et al. Classification and grading of gastritis. The updated Sydney System. International Workshop on the Histopathology of Gastritis, Houston 1994. Am J Surg Pathol 1996;20: 1161-1181.

19 Lazarus M, Munday CJ, Eguchi N, et al. Immunohistochemical localization of microsomal PGE synthase-1 and cyclooxygenases in male mouse reproductive organs. Endocrinology 2002;143:2410-2419.

20 Murakami M, Nakashima K, Kamei D, et al. Cellular prostaglandin E2 production by membrane-bound prostaglandin E synthase-2 via both cyclooxygenases1 and -2. J Biol Chem 2003;278:37937-37947.

21 Lin $\mathrm{H}$, Wada $\mathrm{K}$, Yonezawa $\mathrm{M}$, et al. Tomoregulin ectodomain shedding by proinflammatory cytokines. Life Sci 2003;73:1617-1627.

22 Yamagata K, Matsumura K, Inoue W, et al. Coexpression of microsomal-type prostaglandin E synthase with cyclooxygenase-2 in brain endothelial cells of rats during endotoxins-induced fever. J Neurosci 2001;21: 2669-2677.

23 Jabbour HN, Milne SA, Williams ARW, et al. Expression of COX-2 and PGE synthase and synthesis of PGE2 in endometrial adenocarcinoma: a possible autocrine/paracrine regulation of neoplastic cell function via EP2/EP4 receptors. Br J Cancer 2001;85: 1023-1031.

24 Parent J, Chapdelaine P, Sirois J, et al. Expression of microsomal prostaglandin E synthase in bovine endometrium: coexpression with cyclooxygenase type 2 and regulation by interferon- $\tau$. Endocrinology 2002; 143:2936-2943.

25 Lazarus M, Kubata BK, Eguchi N, et al. Biochemical characterization of mouse microsomal prostaglandin $\mathrm{E}$ synthase- 1 and its colocalization with cyclooxygenase2 in peritoneal macrophages. Arch Biochem Biophys 2002;397:336-341.

26 Han R, Tsui S, Smith T. Up-regulation of prostaglandin E2 synthesis by interleukin-1 $\beta$ in human orbital fibroblasts involves coordinate induction of prosta- glandin-endoperoxide $\mathrm{H}$ synthase-2 and glutathionedependent prostaglandin E2 synthase expression. J Biol Chem 2002;277:16355-16364.

27 Murakami M, Nakatani Y, Tanioka T, et al. Prostaglandin E synthase. Prostaglandins Other Lipid Mediators 2002;68-69:383-399.

28 Claveau D, Sirinyan M, Guay J, et al. Microsomal prostaglandin E synthase-1 is a major terminal synthase that is selectively up-regulated during cyclooxygenase-2-dependent prostaglandin E2 production in the rat adjuvant-induced arthritis model. J Immunol 2003;170:4738-4744.

29 Stichtenoth D, Thoren S, Bian H, et al. Microsomal prostaglandin E synthase is regulated by proinflammatory cytokines and glucocorticoids in primary rheumatoid synovial cells. J Immunol 2001;167:469-474.

30 Kojima F, Naraba H, Sasaki Y, et al. Coexpression of microsomal prostaglandin E synthase with cyclooxygenase-2 in human rheumatoid synovial cells. J Rheumatol 2002;29:1836-1842.

31 Romano M, Ricci V, Memoli A, et al. Helicobacter pylori up-regulates cyclooxygenase-2 mRNA expression and prostaglandin E2 synthesis in MKN 28 gastric mucosal cell in vitro. J Biol Chem 1998;273: 28560-28563.

32 Akhtar M, Cheng Y, Magno RM, et al. Promoter methylation regulates Helicobacter pylori-stimulated cyclooxygenase-2 expression in gastric epithelial cells. Cancer Res 2001;61:2399-2402.

33 McCarthy CJ, Crofford LJ, Greenson J, et al. Cyclooxygenase-2 expression in gastric antral mucosa before and after eradication of Helicobacter pylori infection. Am J Gastroenterol 1999;94:1218-1223.

34 Kimura A, Tsuji S, Tsujii M, et al. Expression of cyclooxygenase-2 and nitrotyrosine in human gastric mucosa before and after Helicobacter pylori eradication. Prostaglandins Leukot Essent Fatty Acids 2000; 63:315-322.

35 Chan FK, To KF, Ng YP, et al. Expression and cellular localization of COX-1 and -2 in Helicobacter pylori gastritis. Aliment Pharmacol Ther 2001;15: 187-193.

36 Nardone G, Rocco A, Vaira D, et al. Expression of COX-2, mPGE-synthase1, MDR-1 (P-gp), and Bcl-XL: a molecular pathway of $H$. pylori-related gastric carcinogenesis. J Pathol 2004;202:305-312.

37 Steinbach G, Lynch PM, Phillips RK, et al. The effect of celecoxib, a cyclooxygenase-2 inhibitor, in familial adenomatous polyposis. N Engl J Med 2000;342: 1946-1952.

38 White WB, Faich G, Borer JS, et al. Cardiovascular thrombotic events in arthritis trials of the cyclooxygenase-2 inhibitor celecoxib. Am J Cardiol 2003;92: $411-418$

39 Lim H, Paria BC, Das SK, et al. Multiple female reproductive failures in cyclooxygenase-2 deficient mice. Cell 1997;91:197-208.

40 Fuson AL, Komlosi P, Unlap TM, et al. Immunolocalization of a microsomal prostaglandin E synthase in rabbit kidney. Am J Physiol Renal Physiol 2003;285: F558-F564. 